\title{
HIGH LATITUDE GALACTIC EMISSION AND THE SEARCH FOR ANISOTROPIES IN THE CBR
}

\author{
S. Masi, P. Calisse, P. de Bernardis, M. De Petris, M. Epifani, \\ M. Gervasi, G. Guarini, F. Melchiorri, G. Moreno, P. Temi \\ Dipartimento di Fisica \\ Università di Roma, Italy
}

The study of the anisotropies of the submillimeter relict radiation (RR) can provide important arguments to select among different theoretical scenarios (Panek and Rudak 1988). However, interstellar dust (ISD) emission is present in this spectral region and its patchy distribution can heavily contaminate anisotropy measurements. For example, the most sensitive measurement of CBR anisotropies has been reported so far in a broad band around $1 \mathrm{~mm}$ (Melchiorri et al. 1981): the detection of anisotropy is statistically very significant, but its cosmological origin is questionable.

As a first step we study the dust emission spectrum. IRAS diffuse emission data at $\lambda=$ $100 \mu \mathrm{m}$ have a non-zero level which is consistent with an instrumental offset of about $2.5 \mathrm{MJy}$ $\mathrm{sr}^{-1}$ plus a $2.5-3 \mathrm{MJy} \mathrm{sr}^{-1}$ true sky emission. This is well described by a $\operatorname{cosec}(b)$ law plus a small residual zodiacal component (de Bernardis et al. 1988). The galactic component at the north galactic pole (NGP) is $I_{\mathrm{v}}=(7.5 \pm 1.5) \times 10^{-14} \mathrm{~W} \mathrm{~cm}^{-2} \mathrm{sr}^{-1} \mathrm{~cm}^{-1}$. Matsumoto et al. (1988) report an absolute measurement of the diffuse emission at $\lambda=262 \mu \mathrm{m}$ at $b=35^{\circ}$. The emission extrapolated to the NGP using the $\operatorname{cosec}(b)$ law is $I_{v}=(5.9 \pm 1.2) \times 10^{-14} \mathrm{~W} \mathrm{~cm}^{-2}$ $\mathrm{sr}^{-1} \mathrm{~cm}^{-1}$. Other measurements come from differential experiments and are indirect estimates of the true emission at the pole. By applying the $\operatorname{cosec}(b)$ law to the data of Halpern et al. (1988) at 5.8, 14.6, 24.0, $33.4 \mathrm{~cm}^{-1}$, we have at the NGP the following signal in $10^{-15} \mathrm{~W} \mathrm{~cm}^{-1} \mathrm{sr}^{-1} \mathrm{~cm}^{-1}: I\left(5.8 \mathrm{~cm}^{-1}\right)=(0.84 \pm 0.44), I\left(14.6 \mathrm{~cm}^{-1}\right)=(3.9 \pm 1.6), I\left(24.0 \mathrm{~cm}^{-1}\right)$ $=(19 \pm 3), I\left(33.4 \mathrm{~cm}^{-1}\right)=(28 \pm 7)$. In the same wavelength region de Bernardis et al. (1984) have detected diffuse emission from ISD with a balloon differential photometer, covering a narrow circle in the sky at $7^{\circ}<b<50^{\circ}$. They did not directly observe the NGP region. In the low latitude regions a rather good correlation between these data and the Halpern data is found. The emission at NGP inferred from extrapolation of these data is about ten times higher than that found by Halpern et al. This can be explained by observing that the scan of de Bernardis et al. covers low latitudes in the anticenter region, where ISD emission is significantly lower than in the center. A simple $\operatorname{cosec}(b)$ law does not take into account this effect: if the same fit is applied to the Halpern data in the same region, the NGP extrapolated emission is overestimated by a factor of 7 . In the following we will assume that the spectrum of high galactic latitude dust follows the best fit to the data reported before: dust temperature $T_{d}=20 \mathrm{~K}$, spectral index of emissivity $\alpha=2$; this simple single temperature fit follows quite closely the data from three completely different experiments over a frequency span of 20 . Also, the high galactic latitude dust is relatively nearby and thus should have very uniform physical properties.

We modeled the spatial distribution of the ISD emission at high galactic latitudes using the IRAS $100 \mu \mathrm{m}$ survey from the all sky maps. In this way, we can monitor galactic emission from dust clouds with temperatures higher than $\sim 20 \mathrm{~K}$. Cold dust emits at longer wavelengths so that our estimates must be taken as lower limits for the true anisotropy of the ISD emission. In Figure 1 we plot the two "clean" regions where ISD emission is within $10 \%$ of the 
TABLE 1-Anisotropy in the ISD Emission in Several Circular Regions ( $10^{\circ}$ Diameter) at High Galactic Latitudes

\begin{tabular}{|c|c|c|c|c|}
\hline \multicolumn{2}{|c|}{ center of reg. } & \multirow{2}{*}{$\begin{array}{c}\text { data } \\
\#\end{array}$} & \multirow{2}{*}{$\begin{array}{c}\text { average } \\
\left(\mathrm{MJy} \mathrm{sr}^{-1}\right)\end{array}$} & \multirow{2}{*}{$\begin{array}{c}\mathrm{rms} \\
\left(\mathrm{MJy} \mathrm{sr}^{-1}\right)\end{array}$} \\
\hline$b^{\circ}$ & $l^{\circ}$ & & & \\
\hline 85 & 100 & 325 & 7.270 & 0.280 \\
\hline 55 & 101 & 316 & 6.197 & 0.172 \\
\hline 55 & 110 & 308 & 6.227 & 0.192 \\
\hline 50 & 100 & 315 & 6.267 & 0.252 \\
\hline 60 & 100 & 315 & 6.252 & 0.217 \\
\hline-60 & 305 & 295 & 6.420 & 0.295 \\
\hline 50 & 200 & 267 & 11.130 & 1.082 \\
\hline
\end{tabular}

Figure 1. IRAS all sky map at $100 \mu \mathrm{m}$ : in the shaded regions the diffuse emission level is within $10 \%$ of the minimum. minimum value. All the measurements of extragalactic background (EGB) anisotropies in the millimeter and submillimeter region must be performed in these "galactic windows." The fluctuations of the IRAS signal in the clean regions is a combination of detector noise and true sky fluctuations. If we use a $5^{\circ}$ diameter beam we have 72 independent data. The rms fluctuation of the data at $100 \mu \mathrm{m}$ is $0.16 \pm 0.01 \mathrm{MJy} \mathrm{sr}^{-1}$ with an expected detector noise of $0.11 \pm 0.02 \mathrm{MJy} \mathrm{sr}^{-1}$; this implies a true sky noise of $0.12 \pm 0.02 \mathrm{MJy} \mathrm{sr}^{-1}$ which is of the order of $5 \%$ of the ISD emission at NGP. We select three frequencies for numerical examples of ISD emission. In $10^{-15} \mathrm{~W} \mathrm{~cm}^{-1} \mathrm{sr}^{-1} \mathrm{~cm}^{-1}$ we have: $I\left(5 \mathrm{~cm}^{-1}\right) \approx 0.63, \quad I\left(10 \mathrm{~cm}^{-1}\right) \approx 1.8$, $I\left(20 \mathrm{~cm}^{-1}\right) \approx 10$. The $100 \mu \mathrm{m}$ fluctuation corresponds to fluctuations in the CBR $(2.75 \mathrm{~K}$ blackbody) of $\Delta T / T$ of the order of $9 \times 10^{-7}$, $2.6 \times 10^{-6}$, and $1.7 \times 10^{-4}$ at the three frequencies listed above. At an angular scale of $0.5^{\circ}$, several circular regions $10^{\circ}$ in diameter centered at different locations have been studied and are compared in Table 1. The minimum rms fluctuation of the $100 \mu \mathrm{m}$ signal is about $0.7 \mathrm{MJy} \mathrm{sr}^{-1}$; this corresponds to a CBR anisotropy of $5.4 \times 10^{-6}, 1.6 \times 10^{-5}$, and $1.0 \times 10^{-3}$ at the frequencies listed above.

For large scale anisotropy measurements (dipole and quadrupole), a large sky coverage is essential. If a best fit is performed on the IRAS data from the clean regions, the dipole anisotropy due to dust emission at $100 \mu \mathrm{m}$ has an amplitude of $0.034 \mathrm{MJy} \mathrm{sr}^{-1}$ (1 $\sigma$ upper limit). Our extrapolation at larger wavelengths gives a fraction of the kinematic dipole anisotropy of the CBR of the order of $2.7 \times 10^{-4}$, $8.0 \times 10^{-4}$, and $5.2 \times 10^{-2}$ at the three frequencies listed above. This means that very accurate dipole measurements are possible in principle; this should be an important test for spectral distortions.

\section{REFERENCES}

de Bernardis, P. et al 1988, Ap. J., 326, 941.

Halpern, M. et al 1988, Ap. J., 332, 596.

Matsumoto, T. et al. 1988, Ap. J., 329, 567.

Melchiorri, F. et al. 1981, Ap. J. (Letters), 250, L1.

Panek, M. and Rudak, B. 1988, MNRAS, 235, 1169. 